\title{
DATABASE CONNECTOR: A TOOL FOR MANIPULATION ON DIFFERENT DATABASES
}

\author{
Alok Bajpai ${ }^{1}$, Hifzan Ahmad ${ }^{2}$, Rohit Mishra ${ }^{3}$ \\ ${ }^{I}$ Department of Computer Science \& Engineering, S.H.I.A.T.S, Allahabad, Uttar Pradesh, India \\ ${ }^{2}$ Department of Computer Science \& Engineering, KNIT, Sultanpur, Uttar Pradesh, India \\ ${ }^{3}$ Department of Computer Science \& Engineering, S.H.I.A.T.S, Allahabad, Uttar Pradesh, India
}

\begin{abstract}
A database is an organized list of facts and information that can easily be accessed, managed, and updated. Database management system is a software application that interacts with the user and the database itself to capture and analyze data. A general-purpose DBMS is designed to allow the definition, creation, querying, update, and administration of databases. There are many well-known DBMSs which include MySQL, MS Access Database, MS SQL Server, Oracle etc. A database is not generally portable across different DBMSs, but different DBMSs can interoperate by using standards such as SQL and ODBC or JDBC to allow a single application to work with more than one DBMS. This paper presents the salient features of developed DataBase Connector tool. The developed tool allows the user to perform DDL (Data Definition Language) and DML (Data Manipulation Language) command on different DBMSs including MS Access Database, SQL Server Database, SQL Express Database, XML Database and Oracle Server Database. The user has no need to have different software application to perform data definition and data manipulation operation on these different DBMSs.
\end{abstract}

Keywords - Database, Data Definition Language (DDL), Data Manipulation Language (DML), Structured Query Language (SQL), Oracle, and Extensible Markup Language (XML).

\section{INTRODUCTION}

A database is an organized list of facts and information that can easily be accessed, managed, and updated. Database management system is a software application that interacts with the user and the database itself to capture and analyze data. A general-purpose DBMS is designed to allow the definition, creation, querying, update, and administration of databases [1]. The primary goal of a DBMS is to provide a way to store and retrieve database information that is both convenient and efficient [2]. There are many well-known DBMSs which include MySQL, MS Access Database, MS SQL Server, Oracle etc. A database is not generally portable across different DBMSs, but different DBMSs can interoperate by using standards such as SQL and ODBC or JDBC to allow a single application to work with more than one DBMS [1]. The user can store their data in any of these databases for the future use. For instance, if the user has some important data but these data are in different database files e.g. MS Access Database, SQL Server Database SQL Express etc. In this case the user has required the software of all these DBMSs; otherwise the user cannot get the data stored in these database files. To overcome this problem, it is required to design and develop a tool which allows the user to perform Data Definition and Data Manipulation operations on these different DBMSs. The proposed and developed DataBase Connector tool is a dotnet (.Net) application that can be run on any platform supporting a dotnet (.Net) framework. This paper presents the salient features of developed DataBase Connector tool. The implementation of DataBase Connector tool is done through Microsoft Visual Studio 9.0.

\section{OVERVIEW OF DATABASE CONNECTOR}

DataBase Connector is very easy-to-use software tool which allow the user to connect with different databases and perform DDL and DML operations on these databases. DataBase Connector tool provide the user to connect with the MS Access Database, SQL Server Database, SQL Express Database, My SQL Database, and Oracle Database. If the user has only database files (such as xyz.mdb or xyz.mdf), the user has required the software of that type of database to open and perform operation on that files, otherwise user can't open the database file.For example, if the user has database.mdb (MS Access database file), the user must need Microsoft Access to perform operations on this file or if the user has database.mdf(MS SQL database file) the user must need MS SQL software to perform operations on this file etc. The designed and developed DataBase Connector tool allowthe user to access all the database files discuss above without the required database software and the user can get the result by executing all type of queries.

\section{FEATURES OF DATABASE CONNECTOR}

The features of the DataBase Connector tool are as follows:

- Allow the user to connect with different databases

- Connectivity with multiple databases using a single tool

- Provides connection with different databases file without database provider software

- Allow user to perform DDL and DML operations on the database by passing simple query

- Allow the user to save query file

- Allow the user to get the report 


\section{WHY USE DATABASE CONNECTOR?}

DataBase Connector tool providesthe facility to the user to connect either with the database server or windows authentication database, but most of the times it is used to connect with the database file. If the user has a database file of each (MS Access, MS SQL, MYSQL and ORACLE) database then the user is required to have data provider for each database type to perform operations and if the user have no data provider ofthe given databases then the user can't open database file.

DataBase Connector tool solves the above problem by providing the facility to the user to connect with the different databases file without having any database provider. The user can perform the DDL and DML operationson the database file by executing simple queries.

\section{IMPLEMENTATION USING MS VISUAL}

\section{STUDIO 9.0}

The implementation of the presented DataBase Connector tool is carried out with Microsoft Visual Studio 9.0 and provides a graphical user interface. It allows the user to select any of the database file which he/she wants to open and perform operation. The window for the user to choose the type of database is illustrated in Fig. 5.1.

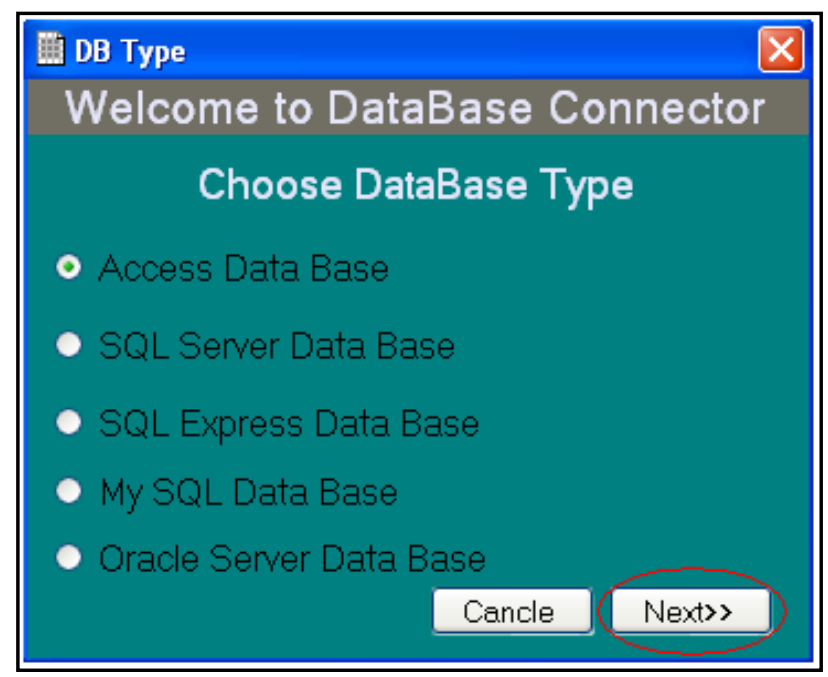

Fig. 5.1: Choose Database Type

The type of database to perform operation is decided by the user. By default Ms Access Database is selected. On clicking the Next button, the user will be redirected to a new window where the user is asked to browse the database file on which he/she wants to perform operations. The new window for browsing the existing database file is shown in Fig. 5.2.

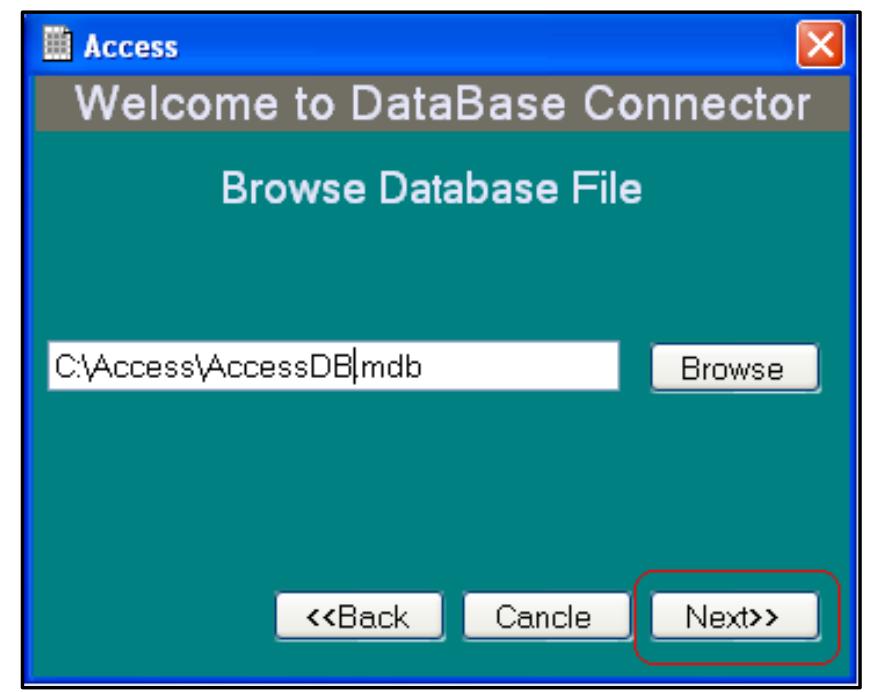

Fig. 5.2: Browse Database File

As shown in Fig. 5.2, the user set the path of the database file on which he/she wants to perform operation. On clicking the Next button the user is redirected to a new window as shown in Fig. 5.3. The newly opened window allows the user to write down the queries to perform DDL and DML operations on the selected database.

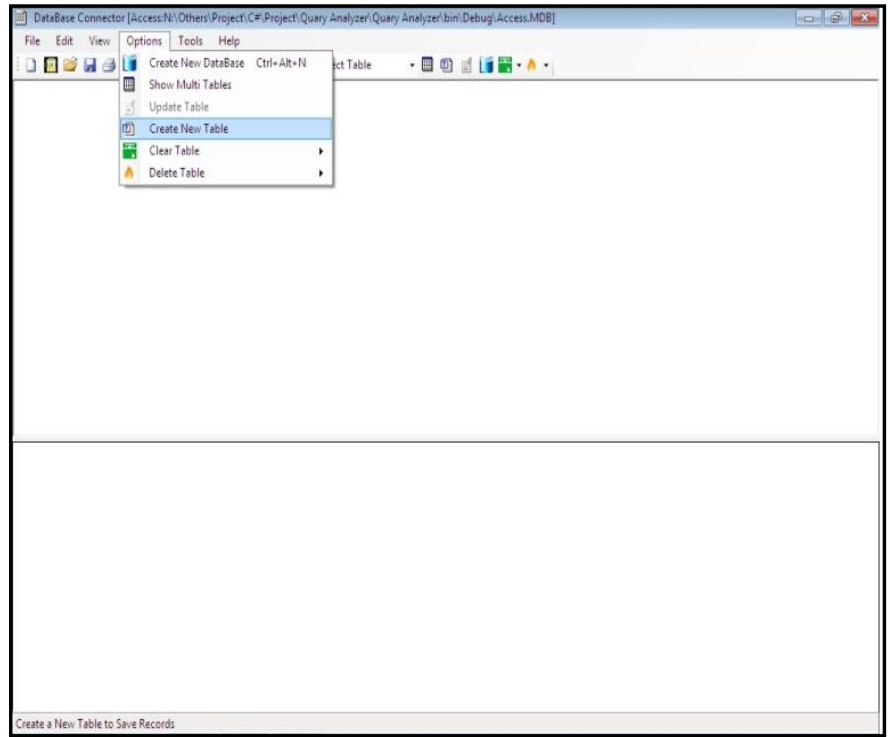

Fig. 5.3: Query Analyzer

In this window as shown in Fig. 5.4, user can write a query to create a new database or new table. The user can also create database or table by using a dialog window.To create a table without writing query, the user can select the Option menu and then select the Create New Table submenu. A new window will be opened for the user to create a table as illustrated in Fig. 5.4. The user can define the fields and their types and the primary key by clicking on the radio button given against each field. On clicking on the Create button, the particular table define by the user with the respected field will be created. The user can also write the query to create a table as shown in Fig. 5.5. 


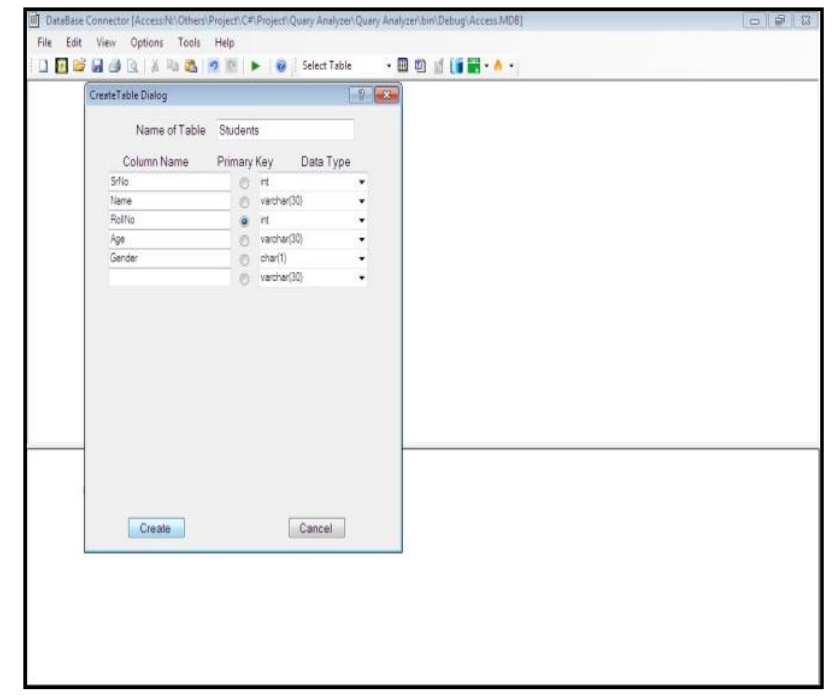

Fig. 5.4: Create Table Dialog

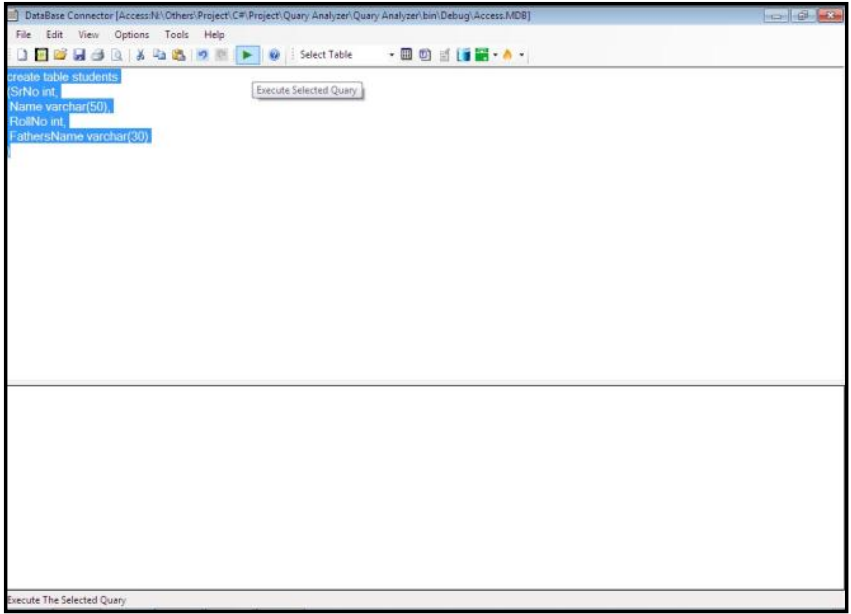

Fig. 5.5: Execute Query to Create Table

For example, the user has written a query to create a table and then perform insertion operation on that table. The user has to select the specific query which he/she wants to execute and then click on the execute button (green button). The selected query will be executed and the output will be shown at the bottom of the window as shown in Fig. 5.6.

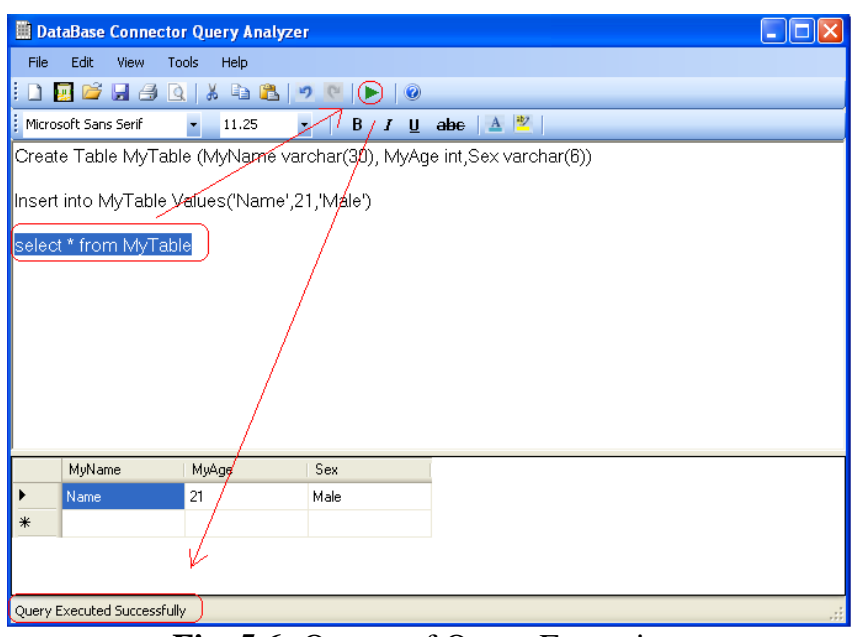

Fig. 5.6: Output of Query Execution
If the user select the SQL Server Database in the choose database type window as shown in Fig. 5.7, the user is redirected to a new window as illustrated in Fig. 5.8, after clicking on the Next button.

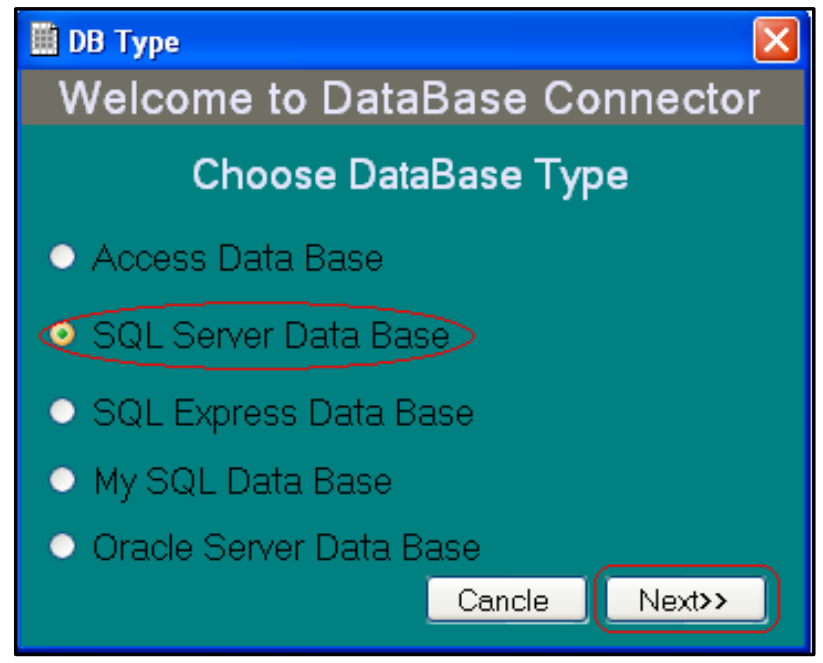

Fig. 5.7: Select SQL Server Database

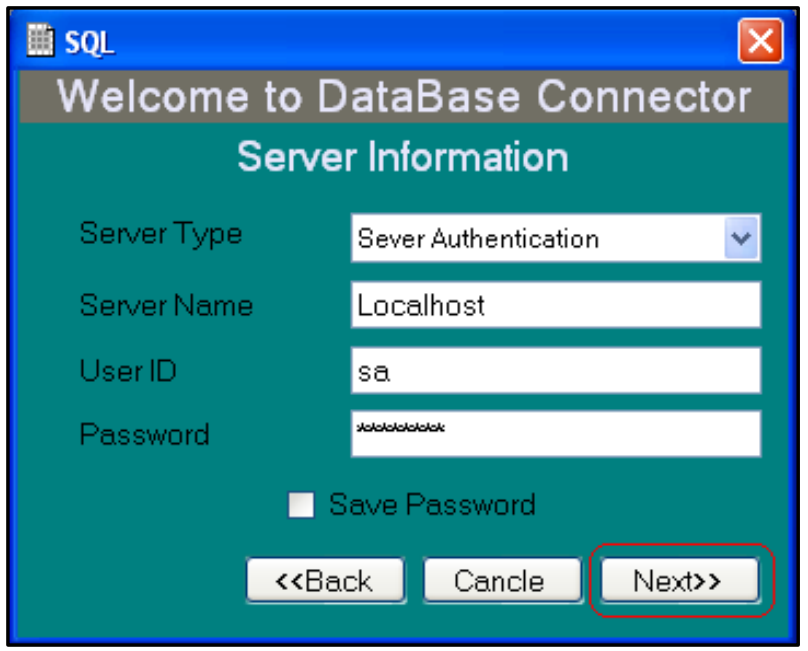

Fig. 5.8: Server Information

As shown in Fig. 5.8, the user has been asked to enter server information. The user will get two options of server types:

- Local Authentication:In local authentication mode, the user does not require login credentials. The user can connect with windows local server without User ID and Password.

- Server Authentication:In server authentication mode, the user has to enter the login credentials to connect with the server. So, a user is required to have the Server Name, User ID and Password.

After giving the details of the server connection when user clicked on the Next button the user is redirected to a new window as shown in Fig. 5.9. The newly opened window asked from the user to select specific database as per the requirement of the user. After selecting the specific database when the user clicked on the Next button, the user is redirected to the window shown in Fig. 5.6. 


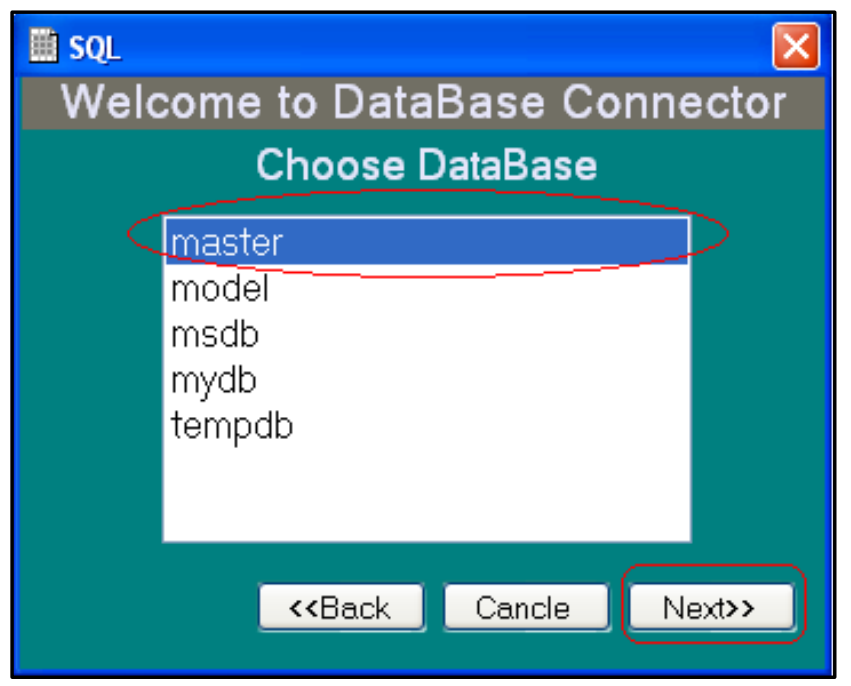

Fig. 5.9: Choose Database

\section{CONCLUSION}

The tools are available to perform operation on specific database files using the required database software of that type. To overcome this type of problem of the user, it is required to design and develop a new type of tool which allows the user to perform DDL and DML operations on different database files by using the single tool. The developed DataBase Connector is very user friendly database tool with graphical user interface. The presented DataBase Connector tool allows the user to perform DDL and DML operations on different database file.

\section{REFERENCES}

[1] "Database - Wikipedia, the free encyclopedia", [Online] Available: http://en.wikipedia.org/wiki/Database, Accessed on: 21Jan-2015

[2] Silberschatz, Korth, Sudarshan (2001), Database System Concepts, 4th Edition 